\title{
Special Issue on Advanced Computational Methods, Systems, and Industrial Engineering Applications
}

\author{
Ha Manh Tran ${ }^{1}$, Vo Nguyen Quoc Bao ${ }^{2}$, Huu-Tue Huynh ${ }^{1}$ \\ ${ }^{1}$ International University, Ho Chi Minh City, Vietnam \\ 2 Posts and Telecommunications Institute of Technology, Ho Chi Minh City, Vietnam
}

Online publication: 10 October 2016, Digital Object Identifier: 10.21553/rev-jec.140

Given the situation of research in Information and Communication Technology in Vietnam, the last few issues of REV Journal on Electronics and Communications published more papers in Algorithms, Intelligent Computation, Pattern Recognition, Information Security, and etc. In pursuing this trend, the editorial team of REV-JEC has decided to dedicate a special issue to topics in these domains.

This special issue consists of selected papers from the International Conference on Advanced Computing and Applications (ACOMP 2015) and the 16th AsiaPacific Industrial Engineering and Management Systems Conference (APIEMS 2015), held in Ho Chi Minh City on November 23-25, 2015, and on December 811, 2015, respectively. The issue aims at seeking strong cross-disciplinary studies connecting the fields of computer science and industrial engineering. Several works that focus on advanced computational methods and industrial engineering applications were selected and invited by the Guest Editor. Each paper submitted to the special issue was reviewed by at least two subject area experts. Final selection decisions were based on reviewers' recommendations and satisfaction with authors' responses.

Five papers were accepted for publication in this special issue. Firstly, Mai Trong Khang, Vu Thanh Nguyen, and Tuan Dinh Le proposed an artificial intelligent approach for improving virus detection. This approach exploits the advantage of the artificial neural network and the artificial immune system to scan large datasets with a high precision rate. Sang Thanh Thi Nguyen and Tuan Thanh Nguyen presented an ontology-based framework for semantic web search. The proposed framework provides an ontology of web documents for seeking the relationship between a web document and a search topic. Tran Ngoc Thinh, Cuong PhamQuoc, Biet Nguyen-Hoang, Chau Tran-Thi, Chien Do-Minh, Quoc Nguyen-Bao, and Nguyen Quoc Tuan took advantage of programmable logic devices for implementation of multiple distributed denial of service countermeasure (DDoS) mechanisms. These devices can quickly adapt and respond to various DDoS attack mechanisms thanks to their high-speed computation capabilities. Khanh Le, Thang Bui and Tho Quan introduced a component-based approach for modeling and verifying wireless sensor networks (WSNs) using Petri Net simulation. This approach significantly reduces the state space required for WSN models in the verification phase. Finally, Cuong Huy Nguyen, Ha Manh Tran, Quy Tran Vu, and Synh Viet Uyen Ha proposed an approach that exploits context-aware data and classification techniques for fault analysis. This approach uses log-event data and context-aware data, including operational data, to construct decision trees which are then utilized for detection of faults in cloud computing systems. We believe that the selected papers focus on interesting research areas that have attracted much attention recently.

We would like to express our gratitude to the editor team, reviewers, and authors of all papers submitted to this Special Issue, for their valuable works and highquality contributions to this publication.

\section{Ha Manh Tran, Guest Editor}

Vo Nguyen Quoc Bao, JEC Editor

Huu-Tue Huynh, JEC Technical Editor-in-Chief

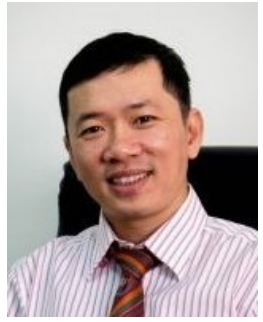

Ha Manh Tran is a lecturer of the School of Computer Science and Engineering at the International University, Vietnam National University, Ho Chi Minh city. He received his master degree of computer science in 2004 from the University of Birmingham, United Kingdom and his doctoral degree of computer science in 2009 from Jacobs University Bremen, Germany. His research interests include distributed computing, big data analytics, information retrieval and network management. 